\title{
Finite Element Analysis (FEA) as a Compiling Course for Undergraduate Students Majoring in Manufacturing Engineering: Case Study
}

\author{
Adham E. Ragab
}

\begin{abstract}
Finite Element Analysis (FEA) is a fundamental technique that is widely used in almost every engineering discipline. Since FEA was introduced during the 1950s, the outcomes of its application in research and development were marvelous. Whether in auto industry, aerospace, ship building construction, etc. researchers from different backgrounds grouped their efforts to enrich and strengthen this technique. Unfortunately, only few engineering colleges teach FEA as an obligatory course for undergraduate junior students. A larger number of colleges offer the FEA course as an elective, while the majority of them limit the course to graduate students. In this paper, the author presents his 4 years of experience in teaching FEA to junior undergraduate students majoring in Manufacturing Engineering at the Canadian International College in Egypt. During those years, the author had built a course that helped students recover material from several previous courses and connect them. The author concluded that, besides enabling senior students to use one of the most powerful techniques in design and development, the FEA course helped them fill the gaps between different engineering subjects and allowed them to retrieve information taken during junior years.
\end{abstract}

Keywords-FEA, Engineering Education, FEA course, manufacturing major, compiling course

\section{INTRODUCTION}

Since the 1950s, finite element method (FEM) has been introduced as a powerful tool in almost all engineering aspects. Applications of this tool in civil engineering, mechanical engineering, electrical engineering and other engineering disciplines are beyond counting. The number of books and papers published in the field is enormous. However, many engineering education institutions consider the subject a fancy topic needed only by graduate students. Based on this theory, very few engineering education institutes offer a course in FEA to senior undergraduate students, and most of them offer the course as an elective.

Teaching FEA was one of the recent research topics in engineering education. The research in this area covered several aspects of interest and showed a great potential for such a course for engineering students [1]-[10]. The course described in this paper was designed and taught at the Canadian International College (CIC) in Egypt for four years by the author. It was an obligatory course offered to senior students majoring in manufacturing engineering. With pre-requisites in advanced calculus, engineering materials and machine design, the student registering in the course was assumed to have basic knowledge in matrix operations, differential equations, computer programming, statics, dynamics, material properties, and stress analysis.

The curriculum of manufacturing major at CIC was a combination of mechanical and industrial courses besides the basic engineering courses. The students studied several pure mechanical courses including machine design, fluid mechanics, thermodynamics and mechanical measurements along with several pure industrial engineering courses including operations research, reliability, feasibility studies and maintenance planning. Manufacturing courses in the curriculum included geometric dimensioning and tolerancing, design for manufacturing, CAD/CAM and three courses in manufacturing processes.

Since CIC was established in 2004, the author was the first instructor to teach the FEA course there. The author had built

Manuscript received Jan. 17, 2016.

Adhem E. Ragab is with the Industrial Engineering Department, King Saud University, Riyadh, Saudi Arabia the course to compile the studied basic and mechanical courses as a whole unit. The goal was to emphasize on previous knowledge including statics, dynamics, material properties, stress analysis and fluid mechanics and to cover some important topics that were not covered in the curriculum including analysis of statically indeterminate structures, heat transfer and vibrations.

While a graduate course in FEA would include a vast amount of theoretical, physical and mathematical details, an undergraduate course would cover only the beginners' level of these issues. The target, again, is to connect pieces of puzzle (gained from curriculum courses) together, rather then, adding new pieces.

\section{Course Structure}

The course was designed to cover both the fundamental theoretical background and the practical applications in several FEA branches. Credit hours for the course were 3 hours (this was actually less than appropriate) with six contact hours per week divided equally between lectures and computer lab. The software selected to cover the practical part of the course was ANSYS academic edition, Comprehensive general purpose FEA software that can deal with almost all FEA applications. For about 40 years, ANSYS was one of the main players in the simulation software market with more than 40,000 customers including some giant companies like Battle, Ford, Airbus, Michelin and Boeing. The ANSYS academic edition allows students to deal with all types of problems within some limitations. Although these limitations do not allow the academic edition to be used in research or industry, they do not hinder the students' ability to tackle all course problems with full capabilities.

The main text book was "Finite Element Analysis: Theory and Application with ANSYS", third edition, written by Professor Dr. Saeed Moaveni [11]. An additional reference book was "Finite Element Procedures", 2nd edition, written by Professor Dr. Bathe, Klaus-Jürgen [12]. The course starts with an introductory section [1 week] followed by a brief review of matrices operations [1 week]. Problem formulation is then covered in details [3 weeks]. Trusses analysis comes next [1 week] followed by axial members, beams and frames [1 week]. Detailed description with deep insight of one-dimensional elements is introduced [2 weeks]. Two-dimensional elements analysis is covered with details on structural and thermal analysis [2 weeks]. Finally, the course briefly touches dynamic analysis [2 week] and fluid mechanics analysis [1 week]. Details of the material covered under these titles are given in the following sections.

\section{FEA INTRODUCTION}

The course started with a brief introduction about the FEA history, applications and software. In this introduction an emphasis was made on the relation between the subject and previous courses in their major. A stress was made on the difference between simple problems they ever faced in their study versus very complex problems that might be solved using FEA. Several video clips were presented to assure the students' understanding of the wide scope and level of complexity that can be achieved within FEA applications. A simple mechanical system problem was then presented (Example 3.1 [12]) to establish a first contact with finite element methodology. The problem, shown in Fig. 1, is then solved using force analysis through free body diagram, a technique that was studied before during elementary statics course. 


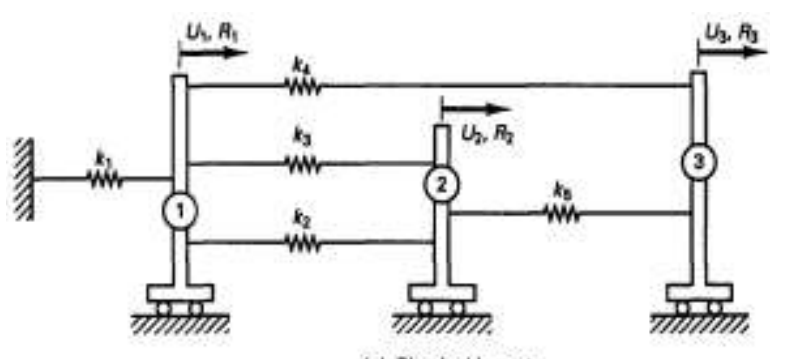

(a) Physical layout

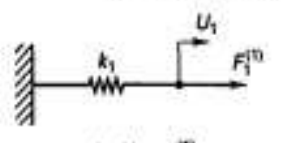

$k_{1} U_{1}=F_{1}^{10}$
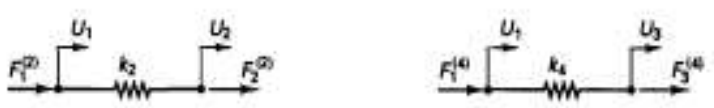

$k_{2}\left[\begin{array}{cc}1 & -1 \\ -1 & 1\end{array}\right]\left[\begin{array}{l}U_{1} \\ U_{2}\end{array}\right] \cdot\left[\begin{array}{c}F_{1}^{12} \\ F_{2}^{12}\end{array}\right]$

$k_{4}\left[\begin{array}{cc}1 & -1 \\ -1 & 1\end{array}\right]\left[\begin{array}{l}U_{1} \\ U_{3}\end{array}\right]=\left[\begin{array}{l}F_{1}^{* i 1} \\ F_{3}^{14}\end{array}\right]$
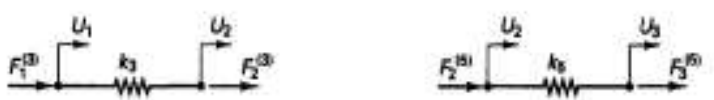

$k_{3}\left[\begin{array}{cc}1 & -1 \\ -1 & 1\end{array}\right]\left[\begin{array}{l}U_{1} \\ U_{2}\end{array}\right]=\left[\begin{array}{c}F_{1}^{13} \\ F_{2}^{13}\end{array}\right]$

(b)

$k_{5}\left[\begin{array}{cc}1 & -1 \\ -1 & 1\end{array}\right]\left[\begin{array}{l}U_{2} \\ U_{3}\end{array}\right]=\left[\begin{array}{l}F_{2}^{15} \\ F_{3}^{15}\end{array}\right]$

Fig. 1. A simple mechanical spring system with force analysis [12]

The analysis of the system results in the well-known governing equation given below.

$$
\mathrm{KU}=\mathrm{R}
$$

Where $\mathrm{K}$ is the system stiffness matrix, $\mathrm{U}$ is the displacement matrix of the rigid carts 1,2 and 3 [U1 U2 U3]T, and R is the force matrix [R1 R2 R3]T. $\mathrm{K}$ is then calculated as follows:

$$
\mathrm{K}=\left[\begin{array}{ccc}
\left(\mathrm{k}_{1}+\mathrm{k}_{2}+\mathrm{k}_{3}+\mathrm{k}_{4}\right) & -\left(\mathrm{k}_{2}+\mathrm{k}_{3}\right) & -\mathrm{k}_{4} \\
-\left(\mathrm{k}_{2}+\mathrm{k}_{3}\right) & \left(\mathrm{k}_{2}+\mathrm{k}_{3}+\mathrm{k}_{5}\right) & -\mathrm{k}_{5} \\
-\mathrm{k}_{4} & -\mathrm{k}_{5} & \left(\mathrm{k}_{4}+\mathrm{k}_{5}\right)
\end{array}\right]
$$

This example proved to be a reasonable beginning of the course that leads the students directly into the core of FEA.

\section{MATH REVIEW}

The main topic in the" math review" section is matrix operations. Most of FEA calculations result in a system of linear equations that is, conveniently, put in a matrix form. The students in this major studied the basics of matrix operation in a sophomore math course. As noted by the instructor, the students did not use matrices in any engineering course. It was essential for the instructor to review the details of basic matrix operations with emphasis on their applications in engineering. The review had to cover several subjects including: basic definitions, matrix addition and subtraction, matrix multiplication, transpose and inverse of a matrix, system of linear equations, eigenvalues and eigenvectors. The most effort exerted in this section was in the area of solving a system of linear equations using the Gauss elimination method. Several examples were given from the text book [11] and some other resources.

Differential equations play an important role in understanding the basics of heat transfer, fluid mechanics and system dynamics. The students, again, studied this course in a pure math course with very limited application in real engineering courses. It was the opportunity in this review to revisit the differential equations subject while stressing its wide applications in the engineering field.

\section{PROBLEM FORMULATION}

Understanding the problem formulation techniques in FEA is the basic step towards intellectual usage of its software packages. The course covered three problem formulation techniques, namely, direct formulation, minimum total potential energy formulation and weighted residual formulation. The latter has four methods included: Collocation method, sub-domain method, Galerkin method and least square method [11].

The first step in teaching direct formulation is using the example given in Fig. 1 as a basis for transferring the students from "force analysis" that is known to them to the idea of "direct formulation" that is new in the course. An additional structural example is given with the finest details to assure the idea of the direct formulation. It is example 1.1 [11] shown in Fig. 2 with its subdivision into elements and nodes.

In this example, the student starts by dividing the bar into elements and nodes and then goes through the direct formulation technique till the calculation of stresses and strains along the bar with non-uniform cross section. The example exposes the student, for the first time, to the idea of meshing. It also allows the instructor to demonstrate a very crucial and useful review on many aspects such as: Force balance, stress and strain definitions, stress strain relationship, modulus of elasticity, poisson's ratio, yield strength, ultimate tensile strength and the major differences between numerical and analytical methods.

The students go one further step that is not included in the example within the text book: Programming the solution of the same bar problem shown in Fig. 2 using MATLAB. Since the students in the course had not studied MATLAB before, it was the instructor's task to teach them the basic principles of programming in MATLAB environment. The matrix-oriented nature of MATLAB makes it a perfect tool to program FEA problems since the student can easily focus on programming the solution procedure instead of spending the time writing codes to conduct matrix operations. Programming such a problem gives the student a very good insight on the FEA and its internal mechanisms.

The student then proceeds to the second example on direct formulation (Example 1.2 [11]) shown in Fig. 3. The course students had not studied heat transfer as a part of their curriculum since the curriculum, as said before, was a combination of mechanical and industrial courses. This example was a good opportunity to introduce the students to the subject of heat transfer with a brief illustration of conduction and convection phenomena and formulas.
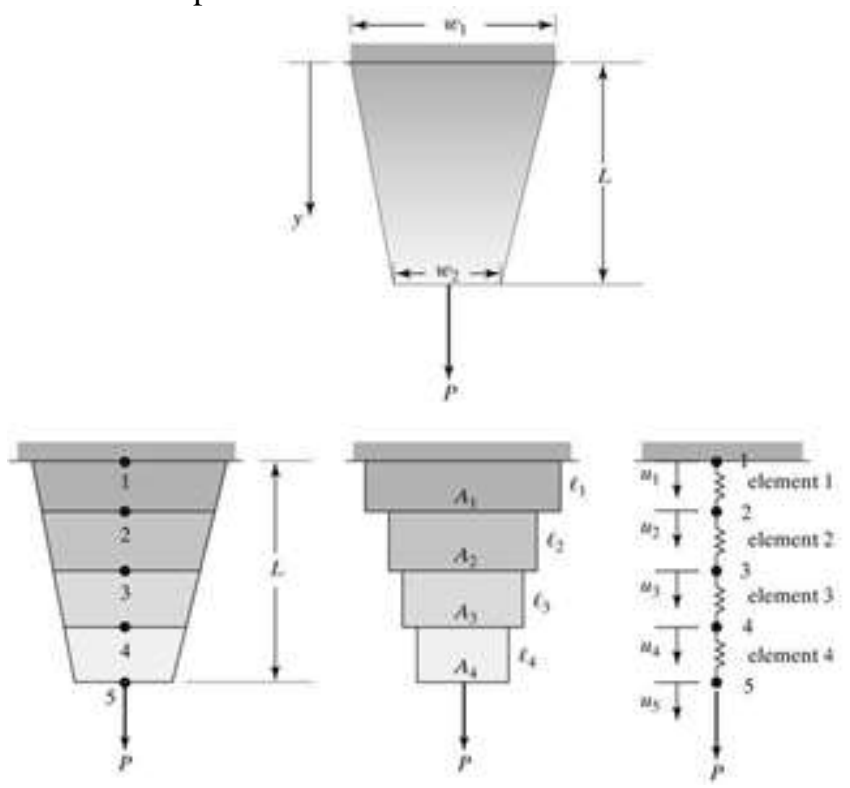

Fig. 2. A bar under axial load with subdivision into elements and nodes
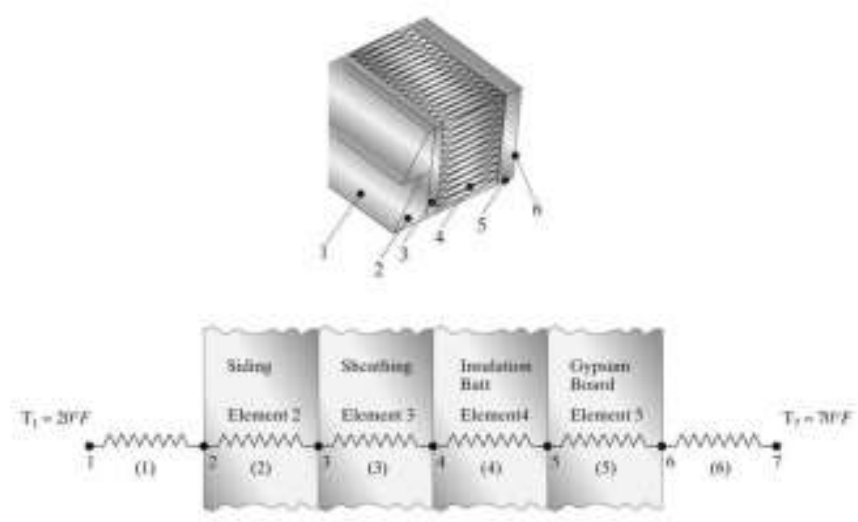

Fig.3. Simple heat transfer problem [11] 
The next step in the course is to use the minimum total potential energy formulation in an example. This technique is tackled through using it to resolve the examples shown in Fig. 1 and Fig. 2. A brief description is then given to the four methods of weighted residual formulations with application on the example shown in Fig. 2.

\section{STRUCTURAL ANALYSIS}

Structural analysis is the main topic in this course. As of the instructor's experience, it is the most appropriate application to convey the FEA concepts and techniques to students. Their familiarity with problems in statics, strength of materials and machine design allows the instructor to focus on the FEA procedures without much distraction in illustrating the problem itself. Also, the mathematics in most of structural analysis problems is simple in nature compared to heat transfer, dynamic or fluid mechanics analysis.

In this course, the structural analysis part begins with truss analysis. The simplicity of the truss analysis comes from the fact that all truss members are two-force members. This means that the internal forces in each truss member are equal and working in opposite directions along the member, as shown in Fig. 4 [11]. This property of truss members allows the students to build the truss FE model easily. Since the students were trained in a previous course to solve truss problems, they were able to compare their solutions using FEA formulation to the graphical or analytical solutions. The example shown in Fig. 5 [11] was used to illustrate this subject.
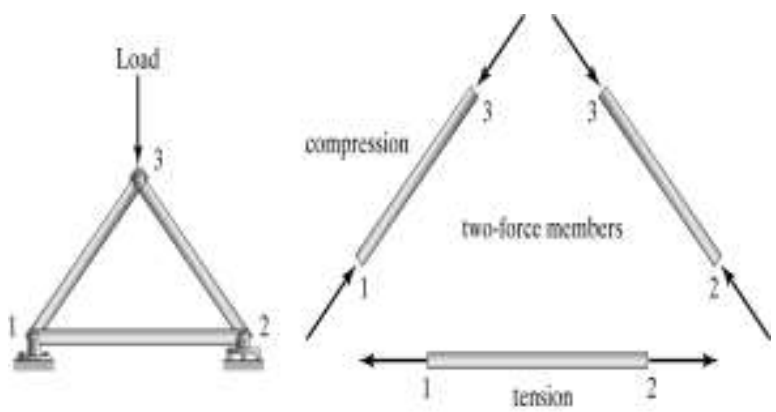

Fig. 4. Simple truss loading [11]

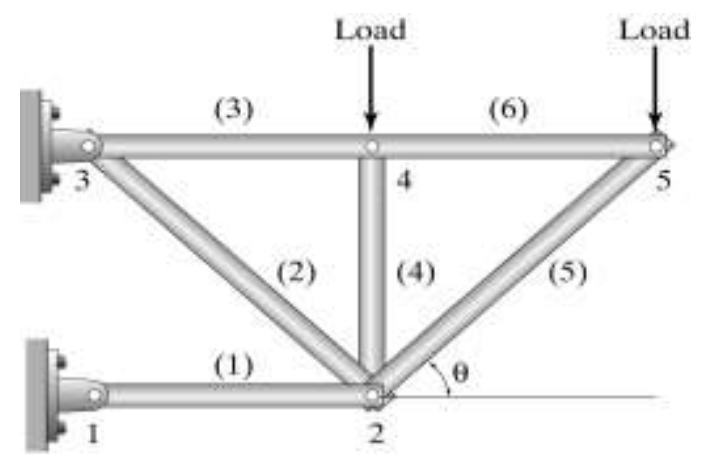

Fig. 5. Statically indeterminate truss [11]

However, considering the simplicity of truss analysis, it was a good opportunity to introduce a complicating element which is the static indeterminacy of structural systems. For the targeted students, it was the first time to deal with statically indeterminate system. The emphasis in this case was on the efficiency of FEA techniques in dealing with statically indeterminate structural systems in the same manner the statically determinate system was dealt with. The example mentioned in the previous paragraph is a statically indeterminate truss.

The example was then solved using ANSYS software. During this ANSYS session, the students had to focus on applying the loads and boundary conditions. The goal was not only to define the loads and boundary conditions in ANSYS, but also, more importantly, to extract the necessary definitions from the structure design. Boundary conditions have a great impact on FEA results; therefore, the student should be aware that their analysis might go completely wrong using inappropriate boundary conditions.

The students then move to the analysis of beams under different types of loadings. In this section the idea of linear and quadratic elements is introduced with relation to shape functions. Understanding the shape functions is important to decide which types of elements to use under different analysis situations. Several examples are introduced in this section to cover its wide range of problems as shown in Fig. 6 - Fig. 8 [11]. These problems are solved manually and using ANSYS.

Structural analysis is revisited during the course to introduce the $2 \mathrm{D}$ and $3 \mathrm{D}$ elements. The emphasis in this section is on the types of elements. More details regarding linear and quadratic elements and their shape functions are illustrated. 2D and 3D problems were solved only using ANSYS due to the extensive calculations needed in a manual solution.

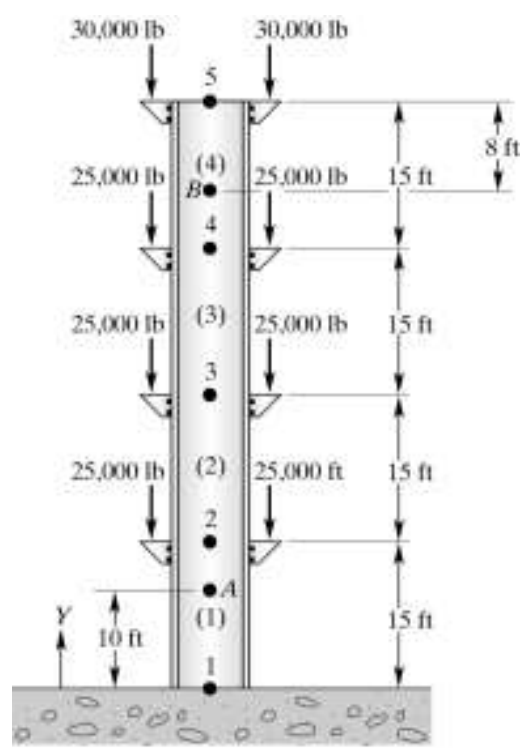

Fig. 6. Column problem [11]

$25,000 \mathrm{~N} / \mathrm{m}$

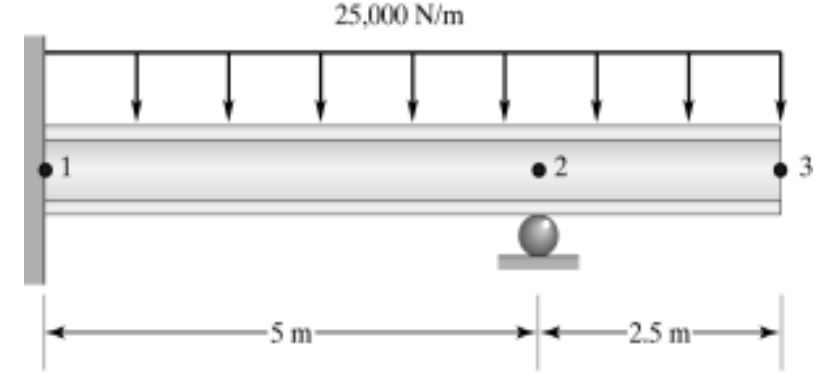

Fig. 7. Statically indeterminate beam problem [11]

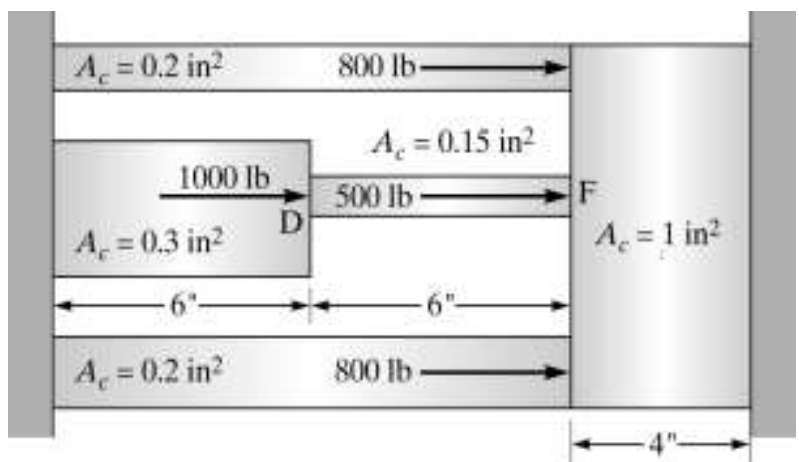

Fig. 8. Structural system with different cross sections [11]

\section{HEAT TRANSFER ANALYSIS}

As mentioned earlier, the students were subjected to the heat transfer analysis in problem formulation section. More details about heat transfer were given with several examples solved manually and using ANSYS. One important problem in the subject is the heat transfer in fins. Equation 2 [11] governs the phenomena. Fig. 9 and Fig. 10 show examples of 1D and 2D heat transfer problems respectively. The first example was solved manually and using ANSYS, while the second example was solved using ANSYS only. 


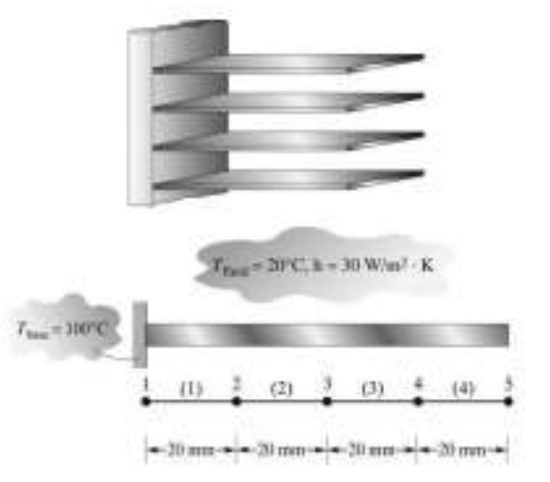

Fig. 9. 1D fin problem [11]

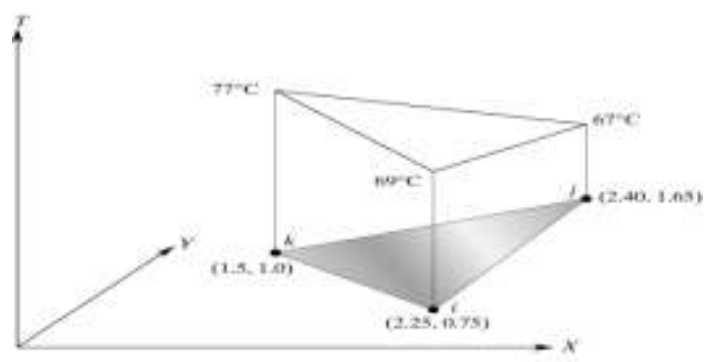

Fig. 10. 2D heat transfer problem [11]

$$
\mathrm{kA} \frac{\mathrm{dT}^{2}}{\mathrm{dx}^{2}}-\mathrm{hpT}+\mathrm{hpT}_{\mathrm{f}}
$$

Where: $\mathrm{k}=$ is the thermal conductivity of the fin material, $\mathrm{A}=$ the cross section area of the fin, $h=$ the convective heat transfer coefficient, $\mathrm{p}=$ the fin perimeter, $\mathrm{Tf}=$ the temperature of the surrounding fluid and $\mathrm{T}=$ the temperature along the fin length.

\section{DYNAMIC ANALYSIS}

This section begins with a general revision of dynamics. The revision covers: The kinematics and kinetics of a particle and the kinematics and kinetics of a rigid body with several examples and practice problems. As the students in this major had not studied a course in vibrations, the instructor chose to proceed with a brief illustration of mechanical system vibrations. The vibration section covered several subjects including: Basic definitions, simple harmonic motion, forced vibration of single degree of freedom systems, multiple degrees of freedom systems and an introduction to Lagrange's equation.

The FEA in this section used Lagrange's equation to formulate the dynamic problem for beams and frames. Fig. 11 shows the two examples that were covered in this section [11]. The first example consists of an aluminum rod with given dimensions and material properties. The student is asked to calculate its natural frequencies. The second example asks for the same requirements, but for a steel frame.

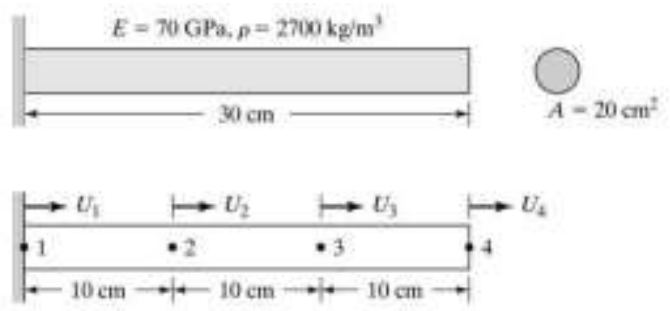

(a)

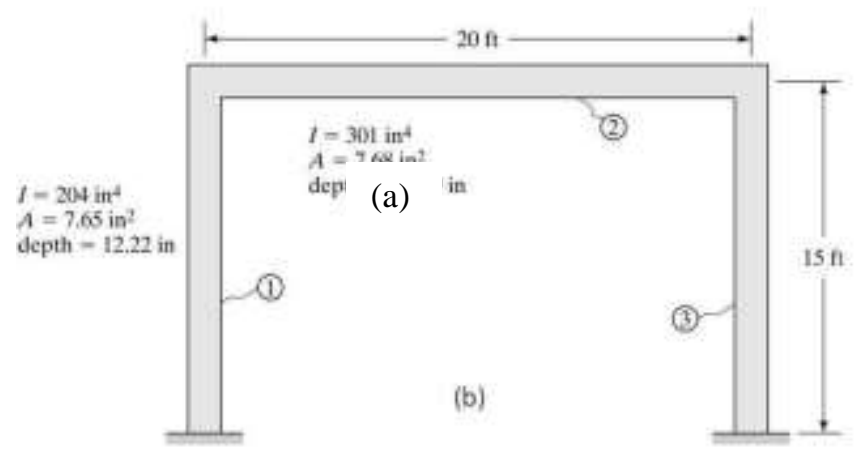

Fig. 11. Two examples on FEA of dynamic systems. a) Simple beam, b) Frame [11]

\section{Fluid MeChanics ANAlysis}

As the students studied a course in fundamentals of fluid mechanics during their sophomore year, only a brief review was needed as an introduction to this section. The review began with fluid kinematics including types of flow and their relation to Reynold's number and continuity equation for incompressible flow in 1D and 2D (Equations 2 and 3). Fluid dynamics, then, was reviewed with emphasis on Bernoulli equation (Equation 4) and its applications in modeling flow through pipes.

$$
\begin{gathered}
A_{l} V_{l}=A_{2} V_{2} \\
\frac{\partial v_{x}}{\partial x}+\frac{\partial v_{y}}{\partial y}=0
\end{gathered}
$$

Where: $\mathrm{A}=$ area and $\mathrm{V}=$ flow speed

$$
\frac{v^{2}}{2}+\frac{p}{\rho}+g \cdot h=\text { constant }
$$

Where: $\mathrm{v}=$ flow speed, $\mathrm{p}=$ pressure, $\rho=$ density, $\mathrm{g}=$ gravity and $\mathrm{h}=$ height.

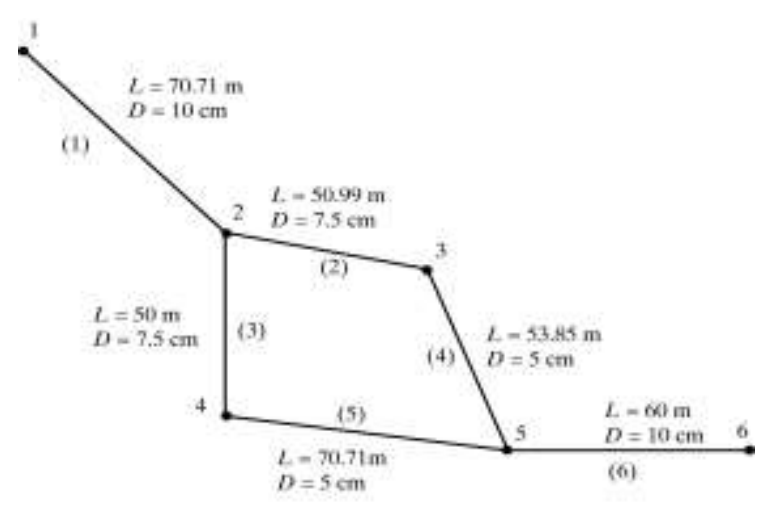

Fig. 12. Flow through pipes example [11]

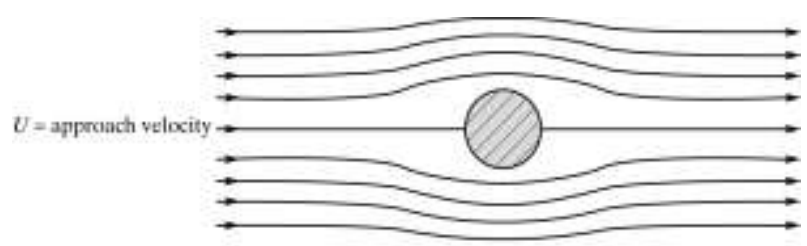

Fig. 13. Ideal flow around a cylinder [11]

The FEA part in this section deals with several aspects. Due to the limited time of the course only two types of problems were illustrated to the students. The first type is flow through pipes with the example shown in Fig. 12. The second type was the ideal flow around a cylinder shown in Fig. 13. The former was solved through formulation and manual solution, while the latter was solved using ANSYS.

\section{CONCLUSION}

The experience of teaching FEA to senior students majoring in Manufacturing Engineering proves the importance and usefulness of such a course. A course in this level might cover several aspects including: FEA problem formulation, structural analysis, heat transfer analysis, dynamic analysis and fluid mechanics analysis. The course works as a compiling course that connects several courses taught in previous years and fills the gaps between taught courses and real world problems. The course also can substitute briefly several missing blocks in the students' curriculum.

A FEA course might be built in different ways to fit different groups of students. Few points should be noticed while designing the course. A brief math review at the beginning of the course allows the students to follow the problems easily. Writing a program to solve few FEA simple problems enhances the students' understanding of the FEA internal mechanisms. The practical part is essential in the course and using commercial software has the advantage of introducing the students to several types of problems conveniently.

One of the common problems noticed during teaching this course, was the great variability between students' background in previous courses. This variability is due to differences in students' levels and the time elapsed between this course and previous background course. To deal with this problem, the 
author had to spend one third of lectures' time (one hour weekly) to cover the required material for the subject in hand. This course needs a long time (about six hours weekly) in both lectures and labs to be covered efficiently.

\section{REFERENCES}

[1] Ural, Ani. "A hands on finite element modeling experience in a multidisciplinary project based freshman course," Computer Applications in Engineering Education, vol. 21 no. 2, pp. 294-299, 2013. http://dx.doi.org/10.1002/cae.20471

[2] Beucke, Karl. "Generalized FEA Framework for Education and Research," Journal of Computing in Civil Engineering, vol. 26, no. 5, pp. 671-679, 2012.

http://dx.doi.org/10.1061/(ASCE)CP.1943-5487.0000154

[3] Lee, Jae Young, and Sung Youll Ahn. "Finite element implementation for computer aided education of structural mechanics: Frame analysis." Computer Applications in Engineering Education, vol. 22, no. 3 , pp. 387-409, 2014

http://dx.doi.org/10.1002/cae.20563

[4] Lee, Jae Young. "Interactive simulation of finite element equation processing for educational purposes," Computer Applications in Engineering Education, vol. 23, no. 2, pp. 157-169, 2015. http://dx.doi.org/10.1002/cae.21586

[5] Jensen, Dan, and Eddy Pramono. "Method for teaching finite elements which combines the advantages of commercial pre-and postprocessing with student written software," Computer Applications in Engineering Education, vol. 6 no. 2, pp. 105-114, 1998. http://dx.doi.org/10.1002/(SICI)1099-0542(1998)6:2<105::AID-CAE6> 3.0.CO;2-H

[6] Mueller, Jr, Donald W. "An introduction to the finite element method using MATLAB," International Journal of Mechanical Engineering Education, vol. 33 no. 3, pp. 260-277, 2005. http://dx.doi.org/10.7227/IJMEE.33.3.8

[7] Steif, Paul S., and Edward Gallagher. "Transitioning students to finite element analysis and improving learning in basic courses," Frontiers in Education, FIE 2004, 34 $4^{\text {th }}$ Annual IEEE, 2004.

[8] Kosasih, P. B. "Learning finite element methods by building applications," International Journal of Mechanical Engineering Education, vol. 38 no. 2, pp. 167-184, 2010 http://dx.doi.org/10.7227/IJMEE.38.2.7

[9] Siswanto, Waluyo Adi, and Agung Setyo Darmawan. "Teaching finite element method of structural line elements assisted by open source freeMat," Research Journal of Applied Sciences, Engineering and Technology, vol. 4, no.10, pp. 1277-1286, 2012

[10] Shaikh, Faiz Uddin Ahmed. "Role of commercial software in teaching finite element analysis at undergraduate level: a case study," Engineering Education, vol. 7, no. 2, pp. 2-6, 2012 http://dx.doi.org/10.11120/ened.2012.07020002

[11] Moaveni, Saeed. Finite element analysis: theory and application with ANSYS. Pearson Education, 3rd edition, 2008. Figures [2-13] are reprinted by permission of Pearson Education, Inc., New York, New York

[12] Bathe, Klaus-Jürgen. Finite element procedures. Pearson Education, 1996. Fig. 1 is reprinted by permission of Prof. Bathe.

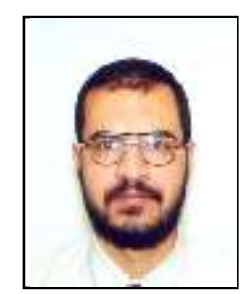

Adham E. Ragab was granted his Ph.D. from Ohio State University, Columbus, Ohio, USA, 2003 in industria engineering. He worked as a researcher at the same university till 2007. Currently he is working at King Saud University, Riyadh, Saudi Arabia as an assistant professor. 